\title{
SUPPRESSION OF MUON BACKGROUNDS GENERATED IN THE ILC BEAM DELIVERY SYSTEM *
}

\author{
A.I. Drozhdin, N.V. Mokhov, N. Nakao ${ }^{\dagger}$, S.I. Striganov, Fermilab, Bavia, IL 60510, USA \\ L. Keller, SLAC, Stanford, CA 94025, USA
}

\begin{abstract}
Muon background suppression at the ILC collider detectors was studied by MARS15 Monte Carlo simulation with a detailed description of the ILC BDS beam line and tunnel of 1.6-km length. Muon suppressions of about $1 / 5$ and $1 / 50$ were obtained for the donut- and wall-shape muon-spoilers, respectively.
\end{abstract}

\section{INTRODUCTION}

Particle fluxes generated in the interactions of a beam halo with the collimators in the ILC Beam Delivery System (BDS) can exceed tolerable levels for the collider detectors and create hostile radiation environment in the interaction region (IR hall). Thorough analysis of the BDS model, beam loss patterns, driving geometry factors and physics processes along with verification of the simulation codes were performed for the current ILC BDS layout with $250-\mathrm{GeV}$ electron and positron beams crossing at $14 \mathrm{mrad}$ with a push-pull detector option.

Suppression of muon background at the IR hall is one of the most important issues for the collider detector performance[1]. Since ILC provides $11 \mathrm{MW}$ electron and position beams, $0.1 \%$ beam loss at BDS causes serious problems for detector background and radiation safety. Using MARS15 code, muon flux at the IR hall was simulated, and muon background suppression were studied with two types of muon-spoiler.

\section{MARS15 MONTE CARLO SIMULATION}

Simulations by the electro-magnetic hadron-cascade transport code, MARS15[2] were performed to obtain spatial distributions of primary and secondary particles in the BDS beam line, tunnel, and IR hall.

\section{Source Term}

For the source term of MARS15 simulation beam halo of $250 \mathrm{GeV}$ positron beam in the ILC BDS was simulated by the STRUCT code[3]. The beam cleaning philosophy is to use large-aperture magnets and collimate the beam at largest possible amplitudes to avoid excessive production of muons. From other side, the synchrotron radiation photons should pass freely through the aperture of IP elements to eliminate photon backgrounds. This requires to collimate halo at $8 \sigma_{x}$ and $65 \sigma_{y}$ in the $8.6-\mathrm{mm}$ thick copper primary-collimators SP2 at s=-1483m and SP4 at

\footnotetext{
*Work supported by Fermi Research Alliance, LLC, under contract No. DE-AC02-07CH11359 with the U.S. Department of Energy.

†nakao@fnal.gov
}

$\mathrm{s}=-1286 \mathrm{~m}$, and in the 35.6-mm thick titanium momentum primary-collimator SPEX at $\Delta p / p=0.0067$ at s=-990m.

Fig.1 shows beam halo particle hits at the three primary collimators SP2, SP4 and SPEX with intensity fractions of $35.7 \%, 42.4 \%$ and $21.9 \%$, respectively. The scraping rate is $0.1 \%$ of total beam intensity, i.e. $2.82 \times 10^{11} \mathrm{sec}^{-1}$.

\section{Beam Loss Distribution}

Beam loss distributions for the above source term along the BDS are shown in Fig.2. Beam loss by secondary $\gamma$ and $\mathrm{e}^{+}$by STRUCT and MARS15 are compared. Agreement is within a factor of 2 . Total energy deposition distribution by MARS15 is compared with that prepared as a source term for the MUCARLO[4] + TURTLE[5] Monte Carlo codes with an approximated method since this combination does not treat photons. The two results agree also within a factor of 2 .



Figure 1: Profiles of the positron beam halo at the three primary collimators by STRUCT.


Figure 2: Halo beam loss along the BDS beam line. 


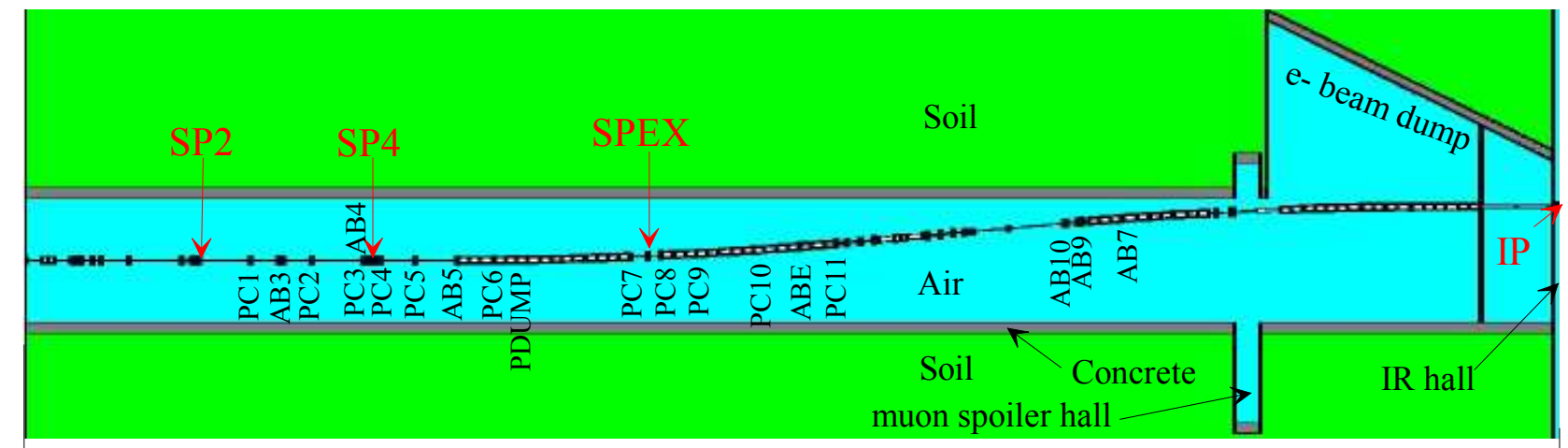

$-1.663 \mathrm{~km}$ from IP

Figure 3: A top view of the BDS tunnel with the entire beam line described in MARS15 simulation. Three beamloss locations at primary collimators(SP2, SP4, SPEX) are shown. The locations of protection collimators(PC) and absorbers $(\mathrm{AB})$ are also shown.

\section{Geometry}

The BDS beam line from $1.663 \mathrm{~km}$ upstream of the interaction point(IP) was described using MAD-MARS Beam Line Builder (MMBLB)[6]. Fig.3 shows a top view of the BDS tunnel with an entire region of the positron beam line in MARS simulation. The IR hall and electron beam dump line are described at $-10 \mathrm{~m}$ and $-320 \mathrm{~m}$ upstream from the IP. Generally, the tunnel cross section is a cylindrical shape with an inner radius of $2 \mathrm{~m}$. Concrete tunnel is 30 -cm thick.

Beam line details are shown in Fig.3. The beam line consists of beam pipes, bending magnets, quadrupole magnets, primary collimators(SP), copper protection collimators(PC, 214mm thick) and copper absorbers(AB, 105$429 \mathrm{~mm}$ thick). The beam-line height is $75 \mathrm{~cm}$ from the floor $(57.29 \mathrm{~cm}$ below the tunnel-cylinder center) in the entire region. The orbit at $-1.663 \mathrm{~km}$ is located in the horizontal center of tunnel.

\section{Muon Spoiler}

Two types of muon-spoilers (wall type and donut type) made of iron with magnetic fields are alternatively used to study suppression of the muon background.

The wall type, as shown in Fig.4, is 5m thick covering overall tunnel cross section located in a muon-spoiler hall upstream of electron beam dump line. Muons are effectively swept after all origins. The wall size is rather large to cover tunnel cross section, that is costly.

Five donut-type muon-spoilers, shown in Fig.5, are located in the straight section after several protection collimators(PCs), which are the locations of the dominant muon origins. Although one muon-spoiler can be smaller in this case, many muon-spoilers are needed to cover most of locations of muon origins.

Although each has pros and cons, muon suppression effectiveness was studied in MARS15 simulation in this work.

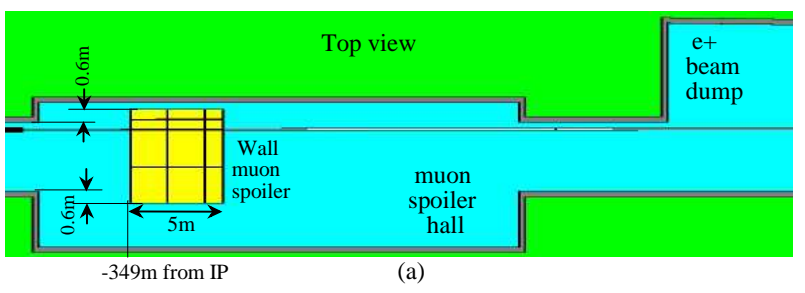

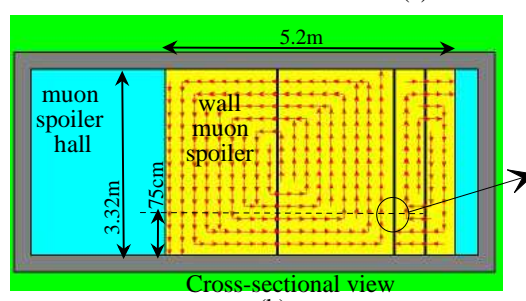

(b)

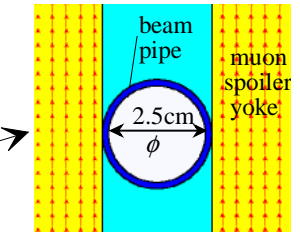

(c)
Figure 4: Top(a) and cross-sectional(b, c) views of the wall muon-spoiler with its dimension and magnetic field image. Beam direction is from back to front of the figures in (b)(c).

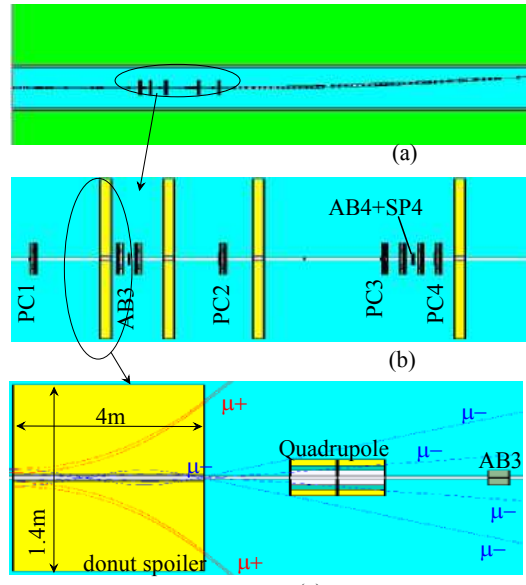

(c)

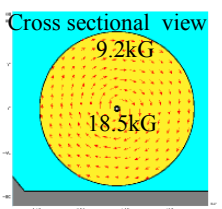

(d)
Figure 5: Top(a,b,c) and cross-sectional(d) views of five donut muon-spoilers and their locations on the beam line. Images of $\mu+$ and $\mu-$ trajectories are shown in (c). 


\section{RESULTS AND DISCUSSIONS}

\section{Muon Distribution}

Fig.6 shows two-dimensional distributions of total muon flux for no, wall and donut muon-spoiler cases. From the figures, most of muons are generated in thick collimators such as PCs and ABs, and the muons bent by dipole and quadrupole magnets are penetrating deeply in soil. From Fig.6(b), the wall muon-spoiler sweeps muons into side soil region quite effectively. From Fig.6(c), on the other hand, although muons generated before the five donut muonspoilers are swept effectively, more donut muon-spoilers are needed also in the bending magnet section where the muon-spoiler can hardly be inserted.

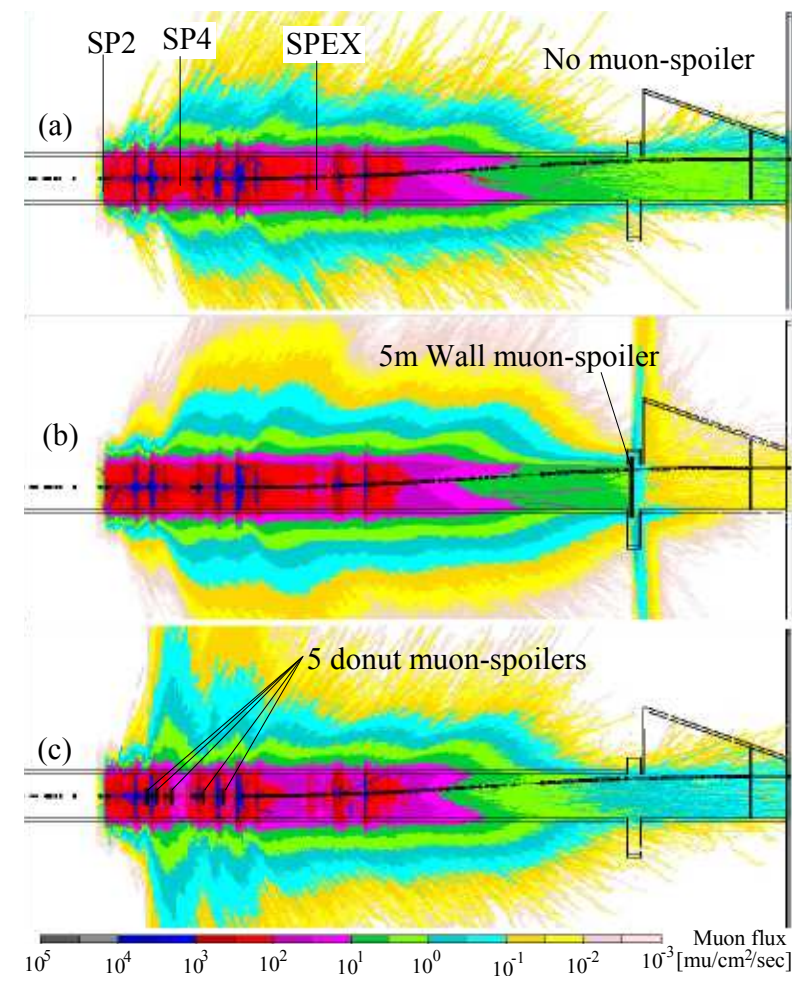

Figure 6: Two dimensional distributions of total muon flux for (a)no, (b)wall and (c)donut muon-spoiler cases.



Figure 7: MUCARLO to MARS ratio for the number of muons at IR at various radii from the beam line for interaction of $250 \mathrm{GeV}$ positrons with individual PCs and ABs.
Table 1: Number of muons per bunch of $2 \times 10^{10}$ positrons by MARS 15 in various radii at IR hall compared among the muon-spoiler types. Ratio to None (\%) is in parenthesis.

\begin{tabular}{l|ccc}
\hline Type & $\mathrm{R}=6.5 \mathrm{~m}$ & $2.5 \mathrm{~m}$ & $2.0 \mathrm{~m}$ \\
\hline None & $14.8(100)$ & $9.10(100)$ & $6.51(100)$ \\
Donut & $2.54(17)$ & $1.59(17)$ & $1.09(17)$ \\
Wall & $0.26(1.8)$ & $0.18(2.0)$ & $0.14(2.2)$ \\
\hline
\end{tabular}

\section{Muon Background at IR hall}

Muon background at various radii from the beam line at the IR hall by MARS15 are given in Table 1. Compared with muons for no muon-spoiler case, suppressions are about $1 / 5$ and 1/50 for donut and wall muon-spoiler, respectively. Muon-background dependence on the muonspoiler type at IR hall can clearly be seen also in Fig.6.

\section{Comparisons between MARS15 and MUCARLO}

Ratios of the muon backgrounds calculated with MARS15 and MUCARLO are shown in Fig.7. MUCARLO does not take into account energy loss straggling. MARS15 results with "turned off" fluctuations become $10 \%$ closer to MUCARLO. A difference between the codes reaches $65 \%$ in the momentum collimation section (AB5 to $\mathrm{ABE}$ ) where only $20 \%$ of the background muons are produced. For the dominating region (PC1 to PC5), the codes agree within $10 \%$.

\section{CONCLUSION}

Muon background suppression at IR hall was simulated by MARS15, and suppressions are about $1 / 5$ and $1 / 50$ for the donut and wall muon-spoilers, respectively. MUCARLO code is in a agreement with MARS15 within 10 to $60 \%$ for $250 \mathrm{GeV}$ positron on protection collimators and absorbers, and it is expected to give a good agreement with MARS15 for the entire simulation.

\section{ACKNOWLEDGEMENTS}

The authors wish to thank Cherrill Spencer (SLAC) for her advices and calculations of the field maps of D- and Q-magnets.

\section{REFERENCES}

[1] D.S. Denisov et al.,JINST (2006); Fermilab-FN-790 (2006).

[2] N.V. Mokhov, "The MARS Code System User's Guide", Fermilab-FN-628 (1995); N.V. Mokhov et al, Fermilab-Conf04/053 (2004); http : //www - ap.fnal.gov/MARS/.

[3] I.S. Baishev et al.,"STRUCT Program User's Reference Manual”, http://www-ap.fnal.gov/users/drozhdin/

[4] L. Keller, SLAC-PUB-6385 (1993)

[5] D.C. Carey et al., SLAC-246 (1980).

[6] M.A. Kostin et al., FERMILAB-FN-0738-rev (2004). 